\title{
Estimates of relativistic electron and proton energy densities in starburst galactic nuclei from radio measurements
}

\author{
Massimo Persic ${ }^{1,2}$ and Yoel Rephaeli ${ }^{3,4}$ \\ 1 INAF-Trieste, via G.B. Tiepolo 11, 34143 Trieste, Italy \\ persic@oats.inaf.it \\ 2 INFN-Trieste, via A. Valerio 2, 34127 Trieste, Italy \\ 3 School of Physics \& Astronomy, Tel-Aviv University, 69978 Tel Aviv, Israel \\ ${ }^{4}$ Center for Astrophysics \& Space Sciences, University of California at San Diego, La Jolla CA 92093, USA \\ Received 13 September 2013 / Accepted 12 May 2014
}

\section{ABSTRACT}

\begin{abstract}
The energy density of energetic protons, $U_{\mathrm{p}}$, in several nearby starburst nuclei (SBNs) has been directly deduced from $\gamma$-ray measurements of the radiative decay of $\pi^{0}$ produced in interactions with ambient protons. Lack of sufficient sensitivity and spatial resolution makes this direct deduction unrealistic in the foreseeable future for even a moderately distant SBN. A more viable indirect method for determining $U_{\mathrm{p}}$ in star-forming galaxies is to use its theoretically based scaling to the energy density of energetic electrons, $U_{\mathrm{e}}$, which can be directly deduced from radio synchrotron and possibly also nonthermal hard X-ray emission. In order to improve the quantitative basis and diagnostic power of this leptonic method we reformulate and clarify its main aspects. Doing so we obtain a basic expression for the ratio $U_{\mathrm{p}} / U_{\mathrm{e}}$ in terms of the proton and electron masses and the power-law indices that characterize the particle spectral distributions in regions where the total particle energy density is at equipartition with that of the mean magnetic field. We also express the field strength and the particle energy density in the equipartition region in terms of the region's size, mean gas density, IR and radio fluxes, and distance from the observer, and determine values of $U_{\mathrm{p}}$ in a sample of nine nearby and local SBNs.
\end{abstract}

Key words. astroparticle physics - ISM: magnetic fields - cosmic rays - galaxies: magnetic fields - galaxies: starburst

\section{Introduction}

Supernovae $(\mathrm{SNe})$ are thought to be the main drivers of particle acceleration via the Fermi-I diffusive shock mechanism (e.g., Gaisser 1990). The radiative yields of relativistic electrons and protons have been measured in the radio to $\mathrm{TeV}$ regions.

Proton interactions with ambient gas protons produce neutral pions $\left(\pi^{0}\right)$, whose decay into $\gamma$ rays is the most significant signature of the main component of cosmic rays (CRs). Measurement of this emission yields the essential ingredient in the energetics of galactic nonthermal particles, and together with synchrotron and Compton emissions by relativistic electrons, allows us to relate diverse phenomena such as star formation (SF) and SN rates, efficiency of particle acceleration, and magnetic field strength. The high SF rates and dense gas in starburst nuclei (SBNs) make these regions prime targets for exploring this relation between stellar and nonthermal quantities.

In a SBN with H-nuclei number density $n$ and volume $V$, the integrated hadronic $\gamma$-ray emission from $\pi^{0}$ decay is

$L_{\geq \epsilon}^{\left[q_{\mathrm{p}}\right]} \sim \int_{V} g_{\geq \epsilon}^{\left[q_{\mathrm{p}}\right]} n U_{\mathrm{p}} \mathrm{d} V \mathrm{~s}^{-1}$

with the integral emissivity $g_{\geq \epsilon}^{\left[q_{\mathrm{p}}\right]}$ measured in units of photon $\mathrm{s}^{-1}(\mathrm{H} \text {-atom })^{-1}\left(\mathrm{eV} / \mathrm{cm}^{3}\right)^{-1}$, and $q_{\mathrm{p}}$ the spectral index of the proton power-law distribution (Drury et al. 1994). The value of $U_{\mathrm{p}}$ can be determined from the measured value of $L_{>\epsilon}^{\left[q_{\rho}\right]}$ if $n(r)$ is known. In steady state the spectro-spatial particle distributions can be calculated by numerically solving a convectiondiffusion equation which includes all the relevant energy losses (e.g., Paglione et al. 1996; Torres 2004). Normalizing the particle distributions based on their measured radiative yields estimates their energy densities. In particular, measurement of the $\pi^{0}$-decay $\gamma$-ray emission is the most direct way to determine $U_{\mathrm{p}}$.

Improving the physical basis for a reliable extraction of the proton energy density, $U_{\mathrm{p}}$, from $\gamma$-ray measurements is well motivated and timely, in light of recent satellite and ground-based observations. These include the detection of three nearby starburst galaxies, NGC 253, NGC 3034 (M 82), and NGC 5945, in the $\mathrm{GeV}$ (Ackermann et al. 2012) and $\mathrm{TeV}$ (Acero et al. 2009; Acciari et al. 2009; Lenain et al. 2010) regions. Measured fluxes from these galaxies agree with earlier theoretical predictions (NGC 253: Domingo-Santamaría \& Torres 2005; Rephaeli et al. 2010; NGC 3034: Persic et al. 2008; de Cea et al. 2009; NGC 4945: Lenain et al. 2010) based on convection-diffusion models for proton and electron propagation and energy losses. While there were appreciable differences in the models treated in those works, predicted values for $U_{\mathrm{p}}$ in the SBNs of the three galaxies were around $250 \mathrm{eV} \mathrm{cm}^{-3}$.

Even though most of the CR energy is in protons, the level of the unbeamed hadronic emission in most SBNs is not high enough to obtain a reliable estimation of $U_{\mathrm{p}}$ from the measurement of hadronic $\gamma$-ray emission. This is the main reason why, with current detector sensitivities, $\gamma$-ray emission was detected (as we just noted) in only three nearby starburst galaxies. It is therefore important to reformulate the leptonic route for estimating particle energy densities, testing its viability in these three starburst galaxies, and applying the insight gained from applications of both methods to these nearby galaxies to improve the precision with which $U_{\mathrm{p}}$ and $U_{\mathrm{e}}$ can be determined in SBNs.

Starting with estimates of the duration of a starburst phase, and of the relevant timescales for particle acceleration, energy 
loss, and advection, we show that particle distributions can be in steady state in a SBN. We continue with the usual assumptions in order to relate the proton and electron densities by charge neutrality, and energy densities through equipartition with the mean magnetic field. Doing so we reformulate the hadronic and leptonic methods for determining particle energy densities from measurements of radio and $\gamma$-ray emission, and refine the expressions for the proton-to-electron density and energy density ratios. We then obtain an expression for the equipartition magnetic field and particle energy densities in a SBN in terms of an assumed (theoretically-based) value of the spectral index of the proton density, and basic measured parameters characterizing the region, i.e., size, gas density, IR luminosity, radio flux, radio spectral index, and distance from the observer.

In Sect. 2 we assess the viability of the standard assumption that particle distributions attain a steady state, and the likelihood of reaching energy equipartition with the mean magnetic field in the SBN. The basic expressions for proton-to-electron (p/e) ratios and for $U_{\mathrm{p}}$ in terms of the electron synchrotron flux are written in Sect. 3. In Sect. 4 these expressions are applied to a sample of nearby and local SBNs. We conclude with a discussion and a summary in Sect. 5.

\section{Energy loss and propagation timescales}

The benchmark timescale for enhanced stellar activity in a SBN, i.e., the duration of the starburst phase, is typically estimated to be $t_{\mathrm{SB}} \sim 10^{8} \mathrm{yr}$. This characteristic time sets the scale for the assessment of temporal non-variability of particle distributions. Specifically, particle densities may attain a steady state if their characteristic acceleration and weighted energy-loss (by collisions and through propagation out of the SBN) timescales are considerably shorter than $t_{\mathrm{SB}}$. If so, and if the acceleration and energy loss timescales are comparable, the particle (spectral) densities are in a steady state.

Acceleration in a SN remnant (SNR) by the Fermi-I process occurs on a timescale $t_{\text {acc }} \equiv E / \dot{E}=(\Delta E / E)^{-1} \Delta t=\beta^{-1} \Delta t=$ $\left(30 / \beta_{0.033}\right) \Delta t \sim 3 \times 10^{5} \mathrm{yr}$, where $\Delta t \sim 10^{4} \mathrm{yr}$ is a typical SNR lifetime, and $\beta_{0.033}$ is the speed of the SN shock in units of $0.033 c=10^{4} \mathrm{~km} \mathrm{~s}^{-1}$. Clearly, particle acceleration to all relevant energies occurs on a relatively short timescale.

Protons lose energy mainly by proton-proton (pp) interactions and escape out of the SBN, on a characteristic timescale $t_{\mathrm{p}}^{-1}=t_{\mathrm{pp}}^{-1}+t_{\mathrm{out}}^{-1}$. The two terms on the r.h.s. are the energyloss timescales for, respectively, pp interactions, $t_{\mathrm{pp}}=\left(\sigma_{\mathrm{pp}} c n\right)^{-1}$ with $\sigma_{\mathrm{pp}}$ the corresponding total cross section, and particle removal, $t_{\text {out }}^{-1}=t_{\text {adv }}^{-1}+t_{\text {diff }}^{-1}$, with $t_{\text {adv }}$ the timescale for advection in a large-scale outflow (i.e., a galactic wind) and $t_{\text {diff }}$ the diffusion timescale. In the energy range $10-10^{5} \mathrm{GeV}$, it is $\sigma_{\mathrm{pp}} \sim$ $50 \mathrm{mb}$, so that $t_{\mathrm{pp}} \sim 2 \times 10^{5}\left(\frac{n}{100 \mathrm{~cm}^{-3}}\right)^{-1}$ yr. For a homogeneous distribution of $\mathrm{SNe}$ in a SBN of radius $r_{\mathrm{s}}$, the advection timescale for transfer of particles out of the disk mid-plane region in a fast $\left(v_{\mathrm{adv}} \sim 1000 \mathrm{~km} \mathrm{~s}^{-1}\right) \mathrm{SB}$-driven wind ${ }^{1}$ is $t_{\mathrm{adv}}=$ $5 \times 10^{4}\left(r_{\mathrm{s}} / 0.2 \mathrm{kpc}\right)\left(v_{\text {out }} / 1000 \mathrm{~km} \mathrm{~s}^{-1}\right)^{-1} \mathrm{yr}$. Particle diffusion is likely to be dominated by random-walk through the tangled magnetic fields in the SBN. In this dense region the field coherence scale is expected to be much smaller than in the disk (where the small-scale cellular structure has a coherence scale $\sim 100 \mathrm{pc}$ ), so scaling to a value of $3 \mathrm{pc}$, the characteristic diffusion time of

\footnotetext{
1 This velocity seems appropriate for NGC 3034, given the terminal outflow velocity of $1600-2200 \mathrm{~km} \mathrm{~s}^{-1}$ deduced by Strickland \& Heckman (2009; see also Chevalier \& Clegg 1985).
}

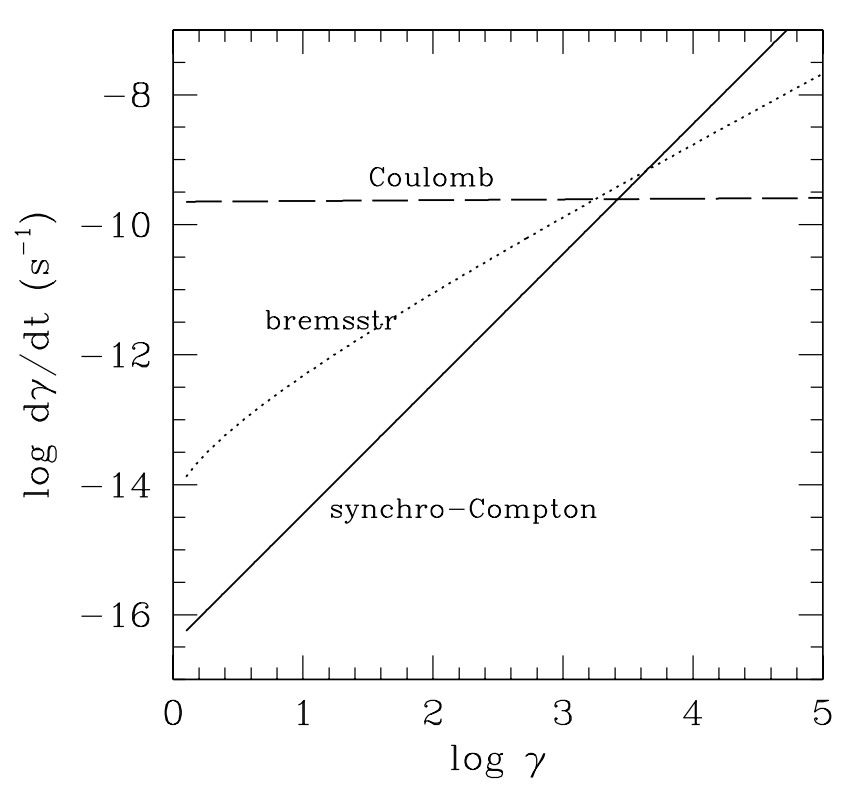

Fig. 1. Energy loss rates of an electron of energy $\gamma m_{\mathrm{e}} c^{2}$ due to Coulomb, bremsstrahlung, and synchrotron-Compton processes in a typical (M 82-like) SBN environment: $B=100 \mu \mathrm{G}, L_{\mathrm{IR}}=10^{44} \mathrm{erg} \mathrm{s}^{-1}$, $r_{\mathrm{s}}=0.2 \mathrm{kpc}, n=100 \mathrm{~cm}^{-3}, n_{\mathrm{e}}=200 \mathrm{~cm}^{-3}$.

protons in the SBN is $t_{\text {diff }}=1.3 \times 10^{5}\left(r_{\mathrm{s}} / 0.2 \mathrm{kpc}\right)^{2}(\lambda / 3 \mathrm{pc})^{-1} \mathrm{yr}$, a value comparable to $t_{\mathrm{adv}}$. From $t_{\mathrm{p}}^{-1}=t_{\mathrm{pp}}^{-1}+t_{\mathrm{out}}^{-1}$, we get $t_{\mathrm{p}} \sim$ $3 \times 10^{4} \mathrm{yr}$ for fiducial parameter values typical for SBNs.

The electron energy loss timescale is determined by Coulomb, bremsstrahlung, synchrotron and Compton processes, and escape out of the SBN, $t_{\mathrm{e}}^{-1}=t_{\mathrm{C}}^{-1}+t_{\mathrm{br}}^{-1}+t_{\mathrm{SC}}^{-1}+t_{\text {out }}^{-1}$, respectively. Limiting the discussion here to electron energies higher than a threshold value $\gamma_{1}$ below which Coulomb losses become relevant (see Sect.4), we can ignore this non-radiative process in the estimation of the weighted mean energy-loss time. The bremsstrahlung cooling time for electrons traveling through ionized gas with number density $n$ is $t_{\mathrm{br}}=4.4 \times 10^{7} n^{-1} \mathrm{yr}$. The synchrotron-Compton time for electrons traversing a region with disordered magnetic field $B$ with energy density $U_{B}=B^{2} /(8 \pi)$, and IR energy density $U_{\mathrm{IR}}=L_{\mathrm{IR}} /\left(4 \pi r^{2} c\right)$, is $t_{\mathrm{SC}}=\frac{3 m_{\mathrm{e}} c}{4 \sigma_{\mathrm{T}}} \gamma^{-1}\left(U_{B}+\right.$ $\left.U_{\mathrm{ph}}\right)^{-1} \simeq \gamma^{-1}\left(U_{B}+U_{\mathrm{ph}}\right)^{-1}$ yr. Under typical SBN conditions, which are fully specified in Sect. 4 (see also Fig. 1), the weighted energy loss time for high energy electrons is $t_{\mathrm{e}} \sim 4.3 \times 10^{4} \mathrm{yr}$ for $\gamma \sim 10^{3}$.

Since typical acceleration and energy-loss times are much shorter than the SB duration, particle spectral densities can attain steady state at levels that are proportional to the respective ratios of the energy-loss time to the acceleration time. More generally, in a state of hydrostatic and virial equilibrium, it is likely that in the minimum-energy configuration, the energy densities of particles and magnetic fields, which are tightly coupled dynamically and energetically in the SN environment, are in energy equipartition (e.g., Longair 1981). Generally, the denser and more radiatively intense the environment, the tighter is the coupling between all degrees of freedom, including nonthermal particles and magnetic fields. Physical processes that couple nonthermal particles and thermal gas are Coulomb interactions (i.e., ionization, electronic excitations, and bremsstrahlung), Compton scattering, and excitation of magnetic turbulence (e.g., Alfvén waves) by non-isotropic particle distributions. Particle coupling to magnetic fields is particularly strong because of the high field strength in a SBN and its disordered morphology, affecting both particle energy distribution and transport properties. 
Table 1. Proton-to-electron energy density ratios, $\kappa$.

\begin{tabular}{ccccccccccccccc}
\hline \hline$q_{\mathrm{p}}$ & $q_{\mathrm{e}}$ & $\kappa$ & $q_{\mathrm{p}}$ & $q_{\mathrm{e}}$ & $\kappa$ & $q_{\mathrm{p}}$ & $q_{\mathrm{e}}$ & $\kappa$ & $q_{\mathrm{p}}$ & $q_{\mathrm{e}}$ & $\kappa$ & $q_{\mathrm{p}}$ & $q_{\mathrm{e}}$ & $\kappa$ \\
\hline 2.0 & 2.0 & $0.258 \mathrm{E}+02$ & 2.1 & 2.0 & $0.984 \mathrm{E}+01$ & 2.2 & 2.0 & $0.418 \mathrm{E}+01$ & 2.3 & 2.0 & $0.197 \mathrm{E}+01$ & 2.4 & 2.0 & $0.101 \mathrm{E}+01$ \\
2.0 & 2.1 & $0.628 \mathrm{E}+02$ & 2.1 & 2.1 & $0.239 \mathrm{E}+02$ & 2.2 & 2.1 & $0.102 \mathrm{E}+02$ & 2.3 & 2.1 & $0.479 \mathrm{E}+01$ & 2.4 & 2.1 & $0.246 \mathrm{E}+01$ \\
2.0 & 2.2 & $0.119 \mathrm{E}+03$ & 2.1 & 2.2 & $0.453 \mathrm{E}+02$ & 2.2 & 2.2 & $0.193 \mathrm{E}+02$ & 2.3 & 2.2 & $0.906 \mathrm{E}+01$ & 2.4 & 2.2 & $0.466 \mathrm{E}+01$ \\
2.0 & 2.3 & $0.189 \mathrm{E}+03$ & 2.1 & 2.3 & $0.720 \mathrm{E}+02$ & 2.2 & 2.3 & $0.306 \mathrm{E}+02$ & 2.3 & 2.3 & $0.144 \mathrm{E}+02$ & 2.4 & 2.3 & $0.740 \mathrm{E}+01$ \\
2.0 & 2.4 & $0.269 \mathrm{E}+03$ & 2.1 & 2.4 & $0.102 \mathrm{E}+03$ & 2.2 & 2.4 & $0.436 \mathrm{E}+02$ & 2.3 & 2.4 & $0.205 \mathrm{E}+02$ & 2.4 & 2.4 & $0.105 \mathrm{E}+02$ \\
2.0 & 2.5 & $0.357 \mathrm{E}+03$ & 2.1 & 2.5 & $0.136 \mathrm{E}+03$ & 2.2 & 2.5 & $0.578 \mathrm{E}+02$ & 2.3 & 2.5 & $0.272 \mathrm{E}+02$ & 2.4 & 2.5 & $0.140 \mathrm{E}+02$ \\
2.0 & 2.6 & $0.451 \mathrm{E}+03$ & 2.1 & 2.6 & $0.172 \mathrm{E}+03$ & 2.2 & 2.6 & $0.731 \mathrm{E}+02$ & 2.3 & 2.6 & $0.344 \mathrm{E}+02$ & 2.4 & 2.6 & $0.177 \mathrm{E}+02$ \\
2.0 & 2.7 & $0.551 \mathrm{E}+03$ & 2.1 & 2.7 & $0.210 \mathrm{E}+03$ & 2.2 & 2.7 & $0.892 \mathrm{E}+02$ & 2.3 & 2.7 & $0.420 \mathrm{E}+02$ & 2.4 & 2.7 & $0.216 \mathrm{E}+02$ \\
2.0 & 2.8 & $0.654 \mathrm{E}+03$ & 2.1 & 2.8 & $0.249 \mathrm{E}+03$ & 2.2 & 2.8 & $0.106 \mathrm{E}+03$ & 2.3 & 2.8 & $0.499 \mathrm{E}+02$ & 2.4 & 2.8 & $0.256 \mathrm{E}+02$ \\
2.0 & 2.9 & $0.760 \mathrm{E}+03$ & 2.1 & 2.9 & $0.289 \mathrm{E}+03$ & 2.2 & 2.9 & $0.123 \mathrm{E}+03$ & 2.3 & 2.9 & $0.579 \mathrm{E}+02$ & 2.4 & 2.9 & $0.298 \mathrm{E}+02$ \\
2.0 & 3.0 & $0.867 \mathrm{E}+03$ & 2.1 & 3.0 & $0.330 \mathrm{E}+03$ & 2.2 & 3.0 & $0.140 \mathrm{E}+03$ & 2.3 & 3.0 & $0.661 \mathrm{E}+02$ & 2.4 & 3.0 & $0.340 \mathrm{E}+02$ \\
\hline
\end{tabular}

Notes. An energy range of $10^{-5} \mathrm{GeV}-10^{5} \mathrm{GeV}$ is assumed.

Under such conditions, particle-field energy equipartition would be expected.

\section{Particle and field energy densities}

In repeated crossings of the shock region, fast electrons and protons in the ambient SNR gas gain energy from their initial fiducial kinetic energy $T_{0} \simeq 10 \mathrm{keV}$ (in the Maxwellian tail) to a very high value, $\mathrm{O}\left(10^{5}\right) \mathrm{GeV}$. In the immediate vicinity of the acceleration sites, and before energy losses substantially modify their initial distributions, the particles' spectral densities are usually assumed to have a power-law (in momentum $p$ ) form, $N_{\mathrm{j}}(p)=N_{0, \mathrm{j}} p^{-q_{\mathrm{j}}}$ with $j=\mathrm{e}, \mathrm{p}$ for, respectively, electrons and protons; in general, $q_{\mathrm{e}} \neq q_{\mathrm{p}}$. If the gas of nonthermal electrons and protons is approximated as an electrically neutral plasma, then

$n_{\mathrm{o}}=\int_{T_{0}}^{\infty} N_{\mathrm{e}}(T) \mathrm{d} T=\int_{T_{0}}^{\infty} N_{\mathrm{p}}(T) \mathrm{d} T$.

The basic energy-momentum relation yields $\mathrm{d} p / \mathrm{d} T=\left(T / c^{2}+\right.$ $m)\left(T^{2} / c^{2}+2 T m\right)^{-1 / 2}$, and using $N_{\mathrm{j}}(T)=N_{\mathrm{j}}[p(T)] \mathrm{d} p / \mathrm{d} T$, an explicit expression for $N_{\mathrm{j}}(T)$ is obtained (e.g., Schlickeiser 2002):

$N_{\mathrm{j}}(T)=\frac{N_{0, \mathrm{j}}}{c^{2}}\left(T+m_{\mathrm{j}} c^{2}\right)\left(\frac{T^{2}}{c^{2}}+2 T m_{\mathrm{j}}\right)^{-\left(q_{\mathrm{j}}+1\right) / 2}$.

The respective normalization can now be obtained by performing the integration in Eq. (2):

$N_{0, \mathrm{j}}=n_{0}\left(q_{\mathrm{j}}-1\right)\left[\frac{T_{0}^{2}}{c^{2}}+2 T_{0} m_{\mathrm{j}}\right]^{\left(q_{\mathrm{j}}-1\right) / 2}$.

The p/e number density ratio, $\zeta=N_{\mathrm{p}}(T) / N_{\mathrm{e}}(T)$, can now be explicitly written

$$
\begin{aligned}
\zeta\left(T ; q_{\mathrm{p}}, q_{\mathrm{e}}\right)= & \frac{\left(q_{\mathrm{p}}-1\right)}{\left(q_{\mathrm{e}}-1\right)} \frac{\left[T_{0}^{2}+2 T_{0} m_{\mathrm{p}} c^{2}\right]^{\frac{q_{\mathrm{p}}-1}{2}}}{\left[T_{0}^{2}+2 T_{0} m_{\mathrm{e}} c^{2}\right]^{\frac{q_{\mathrm{e}}-1}{2}}} \\
& \times \frac{T^{-\frac{\left(q_{\mathrm{p}}+1\right)}{2}}\left(T+m_{\mathrm{p}} c^{2}\right)\left(T+2 m_{\mathrm{p}} c^{2}\right)^{-\frac{q_{\mathrm{p}}+1}{2}}}{T^{-\frac{\left(q_{\mathrm{e}}+1\right)}{2}}\left(T+m_{\mathrm{e}} c^{2}\right)\left(T+2 m_{\mathrm{e}} c^{2}\right)^{-\frac{q_{\mathrm{e}}+1}{2}}} .
\end{aligned}
$$

When it is assumed that $q_{\mathrm{p}}=q_{\mathrm{e}}=q$ (e.g., at injection), simpler limiting expressions for this ratio are obtained (e.g., Schlickeiser 2002):

$\zeta(T ; q)=\left\{\begin{array}{lll}1 & \ldots & T \ll m_{\mathrm{e}} c^{2} \\ \propto\left(\frac{T}{m_{\mathrm{p}} c^{2}}\right)^{\frac{q-1}{2}} & \ldots & m_{\mathrm{e}} c^{2} \ll T \ll m_{\mathrm{p}} c^{2} \\ \left(\frac{m_{\mathrm{p}}}{m_{\mathrm{e}}}\right)^{\frac{q-1}{2}} & \ldots & T \gg m_{\mathrm{p}} c^{2} .\end{array}\right.$

The general expression for the p/e energy density ratio,

$\kappa\left(T_{0} ; q_{\mathrm{p}}, q_{\mathrm{e}}\right)=\frac{\int_{T_{0}}^{\infty} N_{\mathrm{p}}(T) T \mathrm{~d} T}{\int_{T_{0}}^{\infty} N_{\mathrm{e}}(T) T \mathrm{~d} T}$,

can also be written as:

$$
\begin{aligned}
\kappa\left(T_{0} ; q_{\mathrm{p}}, q_{\mathrm{e}}\right)= & \frac{\left(q_{\mathrm{p}}-1\right)}{\left(q_{\mathrm{e}}-1\right)} \frac{\left(T_{0}^{2}+2 T_{0} m_{\mathrm{p}} c^{2}\right)^{\frac{q_{\mathrm{p}}-1}{2}}}{\left(T_{0}^{2}+2 T_{0} m_{\mathrm{e}} c^{2}\right)^{\frac{q_{\mathrm{e}}-1}{2}}} \\
& \times \frac{\int_{T_{0}}^{\infty} T^{-\frac{q_{\mathrm{p}}-1}{2}}\left(T+2 m_{\mathrm{p}} c^{2}\right)^{-\frac{q_{\mathrm{p}}+1}{2}}\left(T+m_{\mathrm{p}} c^{2}\right) \mathrm{d} T}{\int_{T_{0}}^{\infty} T^{-\frac{q_{\mathrm{e}}-1}{2}}\left(T+2 m_{\mathrm{e}} c^{2}\right)^{-\frac{q_{\mathrm{e}}+1}{2}}}\left(T+m_{\mathrm{e}} c^{2}\right) \mathrm{d} T
\end{aligned}
$$

In order to explore the relevant range of values of this ratio, we computed $\kappa$ for several representative values of $q_{\mathrm{p}}$ and $q_{\mathrm{e}}$ (see Table 1). An approximate expression for $\kappa$ can be obtained by considering only proton and electron energies higher than the respective particle mass (where the particle spectra, $N_{\mathrm{j}}(T)$, are single-power-law in energy):

$$
\begin{aligned}
\kappa\left(q_{\mathrm{p}}, q_{\mathrm{e}}\right) \simeq & \frac{\left(q_{\mathrm{p}}-1\right)}{\left(q_{\mathrm{e}}-1\right)} \frac{\left(q_{\mathrm{e}}-2\right)}{\left(q_{\mathrm{p}}-2\right)} \frac{\left(2 T_{0} m_{\mathrm{p}} c^{2}\right)^{\frac{q_{\mathrm{p}}-1}{2}}}{\left(2 T_{0} m_{\mathrm{e}} c^{2}\right)^{\frac{q_{\mathrm{e}}-1}{2}}} \\
& \times \frac{\left(m_{\mathrm{p}} c^{2}\right)^{2-q_{\mathrm{p}}}}{\left(m_{\mathrm{e}} c^{2}\right)^{2-q_{\mathrm{e}}}},
\end{aligned}
$$

which for $q_{\mathrm{p}}=q_{\mathrm{e}}=q$ reduces to

$\kappa(q) \simeq\left(\frac{m_{\mathrm{p}}}{m_{\mathrm{e}}}\right)^{(3-q) / 2}$.

This expression for the energy density ratio (Eq. (10)) is analogous to the high-energy limit of the number density ratio in Eq. (6) (see also Persic \& Rephaeli 2014).

Determining $U_{\mathrm{p}}$ from the theoretically predicted value of $\kappa$ and $U_{\mathrm{e}}$, which is deduced from the measured radio flux, obviously requires knowledge of the mean magnetic field in the 
emitting region, $B$. To overcome this (implied) indeterminacy, the assumption of field and particle energy equipartition is commonly made. In addition, the contribution of secondary electrons (from $\pi^{-}$decay) $)^{2}$ to the (steady state) electron density has to be included.

While the exact form of the particle steady-state spectral density does not generally have a single power-law form (e.g., Rephaeli 1979), the radiative yields are largely by protons and electrons with energies higher than a few Gev, for which Coulomb losses (which flatten the spectral density) are subdominant. In this limit, the total electron spectral density can be approximated by

$N_{\mathrm{e}}(\gamma)=N_{e, 0}(1+\chi) \gamma^{-q_{\mathrm{e}}}$,

where the electron Lorentz factor $\gamma$ is in the range $\gamma_{1} \leq \gamma \leq \gamma_{2}$, $N_{e, 0}$ is a normalization factor of the primary electrons, and $\chi$ is the secondary-to-primary electron ratio. The electron spectral index is $q_{\mathrm{e}} \geq 2$, with the minimal value of 2 corresponding to the strong-shock limit of the Fermi-I acceleration mechanism.

Ignoring the contribution of low-energy electrons with $\gamma<\gamma_{1}$, the electron energy density is $U_{\mathrm{e}} \simeq N_{\mathrm{e}, 0}(1+$ х) $m_{\mathrm{e}} c^{2} \int_{\gamma_{1}}^{\gamma_{2}} \gamma^{1-q_{\mathrm{e}}} \mathrm{d} \gamma$. For $q_{\mathrm{e}}>2$,

$U_{\mathrm{e}}=N_{e, 0}(1+\chi) m_{\mathrm{e}} c^{2} \gamma_{1}^{2-q_{\mathrm{e}}} \frac{\left[1-\left(\gamma_{2} / \gamma_{1}\right)^{2-q_{\mathrm{e}}}\right]}{\left(q_{\mathrm{e}}-2\right)}$.

For a population of electrons described by Eq. (11), traversing a homogeneous magnetic field of strength $B$ that permeates a region of (spherically equivalent) radius $r_{\mathrm{s}}$ located at a distance $d$ from the observer, and emitting a $5 \mathrm{GHz}$ synchrotron radiation flux of $f_{5} \mathrm{Jy}$, the standard synchrotron formula (e.g., Blumenthal $\&$ Gould 1970) yields

$N_{\mathrm{e}, 0}(1+\chi)=1.6 \times 10^{-16} a_{q_{\mathrm{e}}}^{-1} \psi_{5} 1250^{\frac{q_{\mathrm{e}}}{2}} B^{-\frac{q_{\mathrm{e}}+1}{2}}$,

where quantities are expressed in c.g.s. units, the factor $a_{q_{\mathrm{e}}}$ is defined and tabulated (in, e.g., Blumenthal \& Gould 1970), and $\psi_{5} \equiv\left(\frac{r_{\mathrm{s}}}{0.1 \mathrm{kpc}}\right)^{-3}\left(\frac{d}{\mathrm{Mpc}}\right)^{2}\left(\frac{f_{5}}{\mathrm{Jy}}\right)$. From Eqs. (12) and (13) we derive

$$
\begin{aligned}
U_{\mathrm{e}}= & 1.3 \times 10^{-22} 1250^{\frac{q_{\mathrm{e}}}{2}} \psi_{5} B^{-\frac{q_{\mathrm{e}}+1}{2}} \\
& \times \frac{\gamma_{1}^{2-q_{\mathrm{e}}}\left[1-\left(\gamma_{2} / \gamma_{1}\right)^{2-q_{\mathrm{e}}}\right]}{\left(q_{\mathrm{e}}-2\right) a_{q_{\mathrm{e}}}} \mathrm{erg} \mathrm{cm}^{-3} .
\end{aligned}
$$

Since $U_{\mathrm{e}}$ includes both primary and secondary electrons, the rough assumption that both populations can be characterized with nearly the same power-law index ${ }^{3}$ means that the primary

\footnotetext{
2 Secondary positrons (from $\pi^{+}$decay) almost immediately annihilate with thermal electrons.

3 Theoretical expectation is that power-law indices of proton and electron source spectra are nearly identical. Under typical interstellar medium (ISM) conditions, electrons lose energy more efficiently than protons, resulting in a relative steepening of the electron spectrum. Secondary electrons (and positrons, produced in $\pi^{ \pm}$decays following pp collisions) initially have a slightly flatter spectrum (by $\Delta q \simeq 0.2$ ) than the parent proton spectrum for energies $\gtrsim 1 \mathrm{GeV}$, but then their spectrum steepens due to severe energy losses. Detailed numerical models of emission from starburst galaxies show that primary and secondary electrons have roughly similar spectral shapes at energies $\sim 10 \mathrm{MeV}-$ $1 \mathrm{TeV}$ (e.g., Domingo-Santamaría \& Torres 2005; De Cea del Pozo et al. 2009; Rephaeli et al. 2010). Therefore, in the relatively small SBN region, where energetic particles have not yet ventured out too far from their acceleration sites, it is quite reasonable (for our purposes here) to characterize their spectra with the same index. After all, from a practical point of view, both primary and secondary electrons contribute to the main observable upon which we base our analysis - radio emission which is described by a single-index power-law spectrum.
}

electron energy is $U_{\mathrm{e}} /(1+\chi)$. Denoting the primary p/e energy density ratio (see Sect. 3$)$ by $\kappa\left(q_{\mathrm{p}}, q_{\mathrm{e}}\right)$, the proton energy density is

$U_{\mathrm{p}} \simeq \kappa\left(q_{\mathrm{p}}, q_{\mathrm{e}}\right) \frac{U_{\mathrm{e}}}{1+\chi}$.

Since tight coupling is expected in the very dense environment of SBN, particle and magnetic field energy densities can be assumed to be close to equipartition (see Eq. (21)). If so, we can express the field in terms of the total particle energy density; this leads to

$$
\begin{aligned}
U_{\mathrm{p}}= & \frac{2.5 \times 10^{10}}{1+(1+\chi) / \kappa}\left[3.3 \times 10^{-21}\left(1+\frac{\kappa}{1+\chi}\right) \gamma_{1}^{2-q_{\mathrm{e}}}\right. \\
& \left.\times \frac{\left[1-\left(\gamma_{2} / \gamma_{1}\right)^{2-q_{\mathrm{e}}}\right] 1250^{q_{\mathrm{e}} / 2} \psi_{5}}{\left(q_{\mathrm{e}}-2\right) a_{q_{\mathrm{e}}}}\right]^{4 /\left(5+q_{\mathrm{e}}\right)} \mathrm{eV} \mathrm{cm}^{-3}
\end{aligned}
$$

In general, $q_{\mathrm{e}}, q_{\mathrm{p}}, \chi, \gamma_{1}, \gamma_{2}$, and $\kappa$ need to be known (or assumed) in order to compute $U_{\mathrm{p}}$. The value of $q_{\mathrm{e}}$ is readily deduced from measurements of the (nonthermal) radio spectral index, $\alpha$, through the relation $q_{\mathrm{e}}=2 \alpha+1$.

\subsection{Proton spectral index}

The proton spectral index has been measured in the nearby starburst galaxies NGC 253, NGC 3034, and NGC 4945, as $q_{\mathrm{p}} \simeq 2.2$ (Ackermann et al. 2012). As we discuss below, it can be expected that this value also characterizes proton spectra in other starburst galaxies.

Suprathermal particles injected into a supernova shock have a power-law spectrum with index $q=(R+2) /(R-1)$, where $R$ is the shock compression ratio, defined as the downstream to upstream density, $\rho_{\mathrm{d}} / \rho_{\mathrm{u}}$. In an ideal gas, $R=(\gamma+1) /\left[(\gamma-1)+2 / M_{\mathrm{u}}^{2}\right]$, where $\gamma$ is the ratio of the gas specific heats, $M_{\mathrm{u}}=v_{\mathrm{u}} / c_{\mathrm{s}}$ is the shock Mach number, and $c_{\mathrm{S}}$ is the sound speed. Since $c_{\mathrm{s}}=\sqrt{\gamma p_{\mathrm{u}} / \rho_{\mathrm{u}}}$, clearly $M_{\mathrm{u}}=v_{\mathrm{u}} / \sqrt{\gamma k_{B} T_{\mathrm{u}} /\left(\mu m_{\mathrm{p}}\right)}$. The temperature of the upstream medium, $T_{\mathrm{u}}$, is nearly two orders of magnitude higher $\left(T \sim 10^{6-7} \mathrm{~K}\right)$ in SB regions than in more quiescent galactic disks $\left(T \sim 10^{4-5} \mathrm{~K}\right)$ (Heckman \& Lehnert 2000; Fujita et al. 2009). Therefore, for a given shock velocity $v_{\mathrm{u}}$, during the Sedov phase of SNR the Mach number is smaller in the SBN than in the disk, so that the compression ratio of a strong shock in the $\mathrm{SBN}$ is expected to be $R \simeq 3.6$, somewhat lower than the more typical (Galactic) value $R \simeq 4$. This lower value implies an injection index in the range $2.0 \leq q \leq 2.3$ (Fujita et al. 2009).

\subsection{Electron secondary-to-primary ratio}

The secondary-to-primary electron ratio $\chi$ depends on the injection $\mathrm{p} / \mathrm{e}$ number ratio, $\zeta$, and on the gas density, which determines the effectiveness of pp interactions that yield charged and neutral pions. An electron is produced in the decay $\pi^{-} \rightarrow \mu^{-}+\bar{v}_{\mu}$, followed by $\mu^{-} \rightarrow \mathrm{e}^{-}+\bar{v}_{\mathrm{e}}+v_{\mu}$; a positron is produced in the decay $\pi^{+} \rightarrow \mu^{+}+v_{\mu}$, followed by $\mu^{+} \rightarrow \mathrm{e}^{+}+v_{\mathrm{e}}+\bar{v}_{\mu}$. The pp branching ratios are such that in $2 / 3$ of these interactions $\mathrm{e}^{ \pm}$are produced.

The mean free path of an energetic proton for pp interactions in a gas with density $n$ is $\lambda_{\mathrm{pp}}=\left(\sigma_{\mathrm{pp}} n\right)^{-1}$. The pp cross section for protons with kinetic energy of a few $\mathrm{TeV}$ is $\sigma_{\mathrm{pp}} \approx 50 \mathrm{mb}=$ $5 \times 10^{-26} \mathrm{~cm}^{2}$ (Baltrusaitis et al. 1984), so that the probability for a proton to undergo pp interactions during its $3 \mathrm{D}$ random walk through a region of radius $r_{\mathrm{s}}$ is then $\sqrt{3} r_{\mathrm{s}} / \lambda_{\mathrm{pp}}$. Given the injection $\mathrm{p} / \mathrm{e}$ ratio, $\zeta$, and the above branching ratio, the secondaryto-primary electron ratio is

$\chi=\frac{2}{3} \zeta \sqrt{3} r_{\mathrm{s}} n \sigma_{\mathrm{pp}}$. 
Table 2. Starburst galaxies: data and results for high-energy activity.

\begin{tabular}{lcccccccccccccc}
\hline \hline Object & $D_{L}{ }^{1}$ & $R_{\mathrm{SB}}{ }^{2}$ & $h_{\mathrm{SB}}{ }^{3}$ & $f_{5}{ }^{4}$ & $\alpha^{5}$ & $n_{\mathrm{e}}{ }^{6}$ & $L_{\mathrm{IR}}{ }^{7}$ & $M_{\mathrm{SB}}{ }^{8}$ & $\chi^{9}$ & $\kappa^{10}$ & $\gamma_{1}{ }^{11}$ & $B^{12}$ & $U_{\mathrm{p}}{ }^{13}$ & Notes $^{a}$ \\
\hline Arp 220 E & 74.7 & $114^{+}$ & - & 0.08 & 0.70 & $3000^{+}$ & 44.91 & 9.3 & 24 & 48 & 21000 & 155 & 390 & \\
Arp 220 W & 74.7 & $70^{+}$ & - & 0.10 & 0.70 & $3000^{+}$ & 45.08 & 9.1 & 40 & 48 & 15000 & 230 & 730 & \\
Arp 299-A & 43.0 & 140 & $200^{\star}$ & 0.10 & 0.60 & 250 & 44.88 & 9.0 & 8 & 20 & 8700 & 145 & 365 & $=\mathrm{IC} 694$ \\
NGC 253 & 2.5 & 180 & 150 & 1.80 & 0.70 & 100 & 43.62 & 7.7 & 0.3 & 48 & 8300 & 100 & 235 & \\
NGC 3034 & 3.4 & 300 & 200 & 3.70 & 0.71 & 200 & 43.96 & 8.1 & 0.3 & 51 & 6600 & 100 & 250 & $=\mathrm{M} 82$ \\
NGC 3628 & 7.6 & 135 & $200^{\star}$ & .065 & 0.86 & 100 & 43.30 & 7.3 & 0.1 & 120 & 7600 & 65 & 100 & \\
NGC 4945 & 3.7 & 250 & $200^{\star}$ & 2.25 & 0.60 & 300 & 43.72 & 7.4 & 0.1 & 20 & 4700 & 110 & 270 & \\
NGC 5236 & 3.7 & 180 & $200^{\star}$ & 0.75 & 0.80 & 200 & 43.45 & 7.3 & 0.1 & 90 & 5000 & 105 & 260 & $=\mathrm{M} 83$ \\
NGC 6946 & 5.5 & 150 & $200^{\star}$ & .045 & 0.74 & 100 & 43.51 & 7.0 & 0.7 & 60 & 4000 & 65 & 110 & \\
\hline
\end{tabular}

Notes. ${ }^{(1)}$ Distance, in Mpc (from Ackermann et al. 2012). ${ }^{(2,3)}$ Radius and height of (cylindrical) star-forming region, in pc ( $\star:$ assumed; + : spherical). Data are from Torres (2004) and references therein (Arp 220), Sugai et al. (1999, Arp 299-A), Carral et al. (1994, NGC 253), Völk et al. (1996, NGC 3034), Israel et al. (1990, NGC 3628), Brock et al. (1988, NGC 4945), Calzetti et al. (1999, NGC 5236), Schinnerer et al. (2006, NGC 6946). ${ }^{(4,5)}$ Nonthermal 5 GHz flux density, in Jy, and spectral radio index. Data are from Torres (2004, Arp 220), Bondi et al. (2012, Arp 299-A), Rephaeli et al. (2010) and references therein (NGC 253), Klein et al. (1988) and Carlstrom \& Kronberg (1991, NGC 3034), Condon et al. (1982, NGC 3628), Elmouttie et al. (1997, NGC 4945), Sukumar et al. (1987, NGC 5236), Murphy et al. (2011, NGC 6946). ${ }^{(6)}$ Thermal electron density, in $\mathrm{cm}^{-3}$ ( $\star$ : assumed). Data are taken or computed from Yun et al. (2004, Arp 220), Sugai et al. (1999), Zhao et al. (1997, Arp 299-A), Engelbracht et al. (1998, NGC 253), Petuchowski et al. (1994, NGC 3034), Dahlem et al. (1996, NGC 3628), Lipari et al. (1997 and Spoon et al. (2000, NGC 4945), Krabbe et al. (2014, NGC 5236), Engelbracht et al. (1996, NGC 6946). (7) Log of the total IR (8-1000 $\mu \mathrm{m})$ luminosity (erg s${ }^{-1}$; Ackermann et al. 2012; and Charmandaris et al. 2002 for Arp 299-A). ${ }^{(8)}$ Log of ISM mass in the SB (in $M_{\odot}$ ). Data are from Torres (2004, Arp 220), Charmandaris et al. (2002, Arp 299-A), Carral et al. (1994) and Domingo-Santamaría \& Torres 2005 (NGC 253 ), Rickard et al. (1977), Rieke et al. (1980), Weiss et al. (2001, NGC 3034), Israel et al. (1990, NGC 3628), Spoon et al. (2000, NGC 4945), Israel \& Baas (2001, NGC 5236, NGC 6946). (9) Secondary-to-primary electron ratio. ${ }^{(10)} \mathrm{p} / \mathrm{e}$ energy density ratio. (11) Electron Lorentz factor at which Coulomb and synchrotron energy losses are equal. ${ }^{(12)}$ Equipartition magnetic field, in $\mu \mathrm{G}$. ${ }^{(13)}$ Equipartition CR proton energy density, in $\mathrm{eV} \mathrm{cm}^{-3}$. (a) Arp $220 \mathrm{E}$ and W denote, respectively, the east and west extreme starbursts embedded in Arp 220's molecular disk (see Torres 2004 and references therein). NGC 4945 hosts a Seyfert-1 nucleus, but its high-energy $\gamma$ ray emission is dominated by the SBN (Spoon et al. 2000; Lenain et al. 2010).

In a typical SBN with $r_{\mathrm{s}}=0.2 \mathrm{kpc}, n=200 \mathrm{~cm}^{-3}$, and $q=2.2-$ 2.3), estimated values of $\chi$ are $\sim 0.6-1$, in agreement with results from more detailed numerical models (e.g., Paglione et al. 1996; Domingo-Santamaría \& Torres 2005; De Cea et al. 2009; Rephaeli et al. 2010).

\subsection{Equipartition magnetic field}

As we have already noted, it is quite likely that in their equilibrium minimum-energy configuration particles and magnetic fields, which are tightly coupled dynamically and energetically in the SN environment, are in energy equipartition. We assume that equipartition is indeed attained during the starburst phase, and use it to determine the mean magnetic field in the SBN region. The starting point is an estimate of the electron energy density from the measured radio flux, which we obtain by integrating the electron spectral energy density over the interval $\left[\gamma_{1}, \gamma_{2}\right]$.

For consistency with the assumed power-law form of the electron spectral density, we take the low-energy limit $\gamma_{1}$ to be the value of the Lorentz factor at which the sum of the Coulomb (or electronic excitation, in ionized gas) and bremsstrahlung loss rates equals the synchrotron-Compton loss rate. This is also based on the fact that even for the relatively high values of the magnetic field in SBN, the measured radio emission (upon which our normalization of the electron density is based) samples electrons with $\gamma>10^{3}$.

The Coulomb (electronic excitation) loss rate (e.g., Rephaeli 1979) is

$b_{0}=-\dot{\gamma}_{\mathrm{c}} \simeq 1.2 \times 10^{-12} n_{\mathrm{e}}\left[1.0+\frac{\ln \left(\gamma / n_{\mathrm{e}}\right)}{84}\right] \mathrm{s}^{-1}$, where $n_{\mathrm{e}}$ is the (thermal) electron number density.

The closely related bremsstrahlung loss rate for a $\mathrm{H}+\mathrm{He}$ plasma with solar abundances, that also includes the contribution of e-e scatterings, is (Gould 1975)

$$
b_{1}=-\dot{\gamma}_{\mathrm{b}} \simeq \begin{cases}1.78 \times 10^{-16} n \gamma[\ln (\gamma)+0.36] \mathrm{s}^{-1} & \text { ionized } \\ 9.44 \times 10^{-16} n \gamma \mathrm{s}^{-1} & \text { neutral },\end{cases}
$$

where $n$ denotes the number density of H-nuclei. The reported expression for neutral plasma holds for $\gamma \geq 100$; at lower $\gamma$ the ionized and neutral cases essentially overlap (Gould 1975).

The higher order (in $\gamma$ ) synchrotron-Compton loss rate (e.g., Blumenthal \& Gould 1970) is

$b_{2}=-\dot{\gamma}_{\mathrm{SC}}=1.3 \times 10^{-9} \gamma^{2}\left(B^{2}+8 \pi \rho_{\mathrm{IR}}\right) \mathrm{s}^{-1}$,

where $\rho_{\mathrm{IR}}$ is the energy density of the (dominant) IR radiation field in the SBN region.

Equating the sum of the first two loss rates with the latter yields an estimate of $\gamma_{1}$. In Fig. 1 we display the energy-loss rates, expressed in Eqs. (18)-(20), for typical SBN parameters. In our numerical estimates (see Table 2) we use the second of Eq. (19) for the mostly-neutral SBNs in Arp 220 and Arp 299-A, and the first for the other, mostly-ionized SBNs.

Whereas the dependence of the electron energy density on $\gamma_{1}$ is appreciable, since $\gamma_{2}>10^{5}$ the exact value of the upper end of the $\gamma$ integral is of little significance for the range of values of $q_{\mathrm{e}}$ of interest here; in our calculations we take $\gamma_{2}=10^{5}$.

With both $U_{\mathrm{e}}$ and $U_{\mathrm{p}}$ determined, the mean field strength is deduced from

$\frac{B^{2}}{8 \pi}=\eta\left(U_{\mathrm{p}}+U_{\mathrm{e}}\right)$ 
where $\eta=1$ in equipartition, but somewhat lower, $\eta=3 / 4$, in strictly minimum-energy configuration (Longair 1981) ${ }^{4}$. In terms of the particle p/e energy density ratio, $\kappa$, the particle-field coupling condition is $B^{2} / 8 \pi=\eta U_{\mathrm{p}}[1+(1+\chi) / \kappa]$, so that

$$
\begin{aligned}
B= & \sqrt{\eta}\left[3.3 \times 10^{-21}\left(1+\frac{\kappa}{1+\chi}\right) 1250^{q_{\mathrm{e}} / 2} \psi_{5}\right. \\
& \left.\times \gamma_{1}^{2-q_{\mathrm{e}}} \frac{\left[1-\left(\gamma_{2} / \gamma_{1}\right)^{2-q_{\mathrm{e}}}\right]}{\left(q_{\mathrm{e}}-2\right) a_{q_{\mathrm{e}}}}\right]^{2 /\left(5+q_{\mathrm{e}}\right)} \mathrm{G} .
\end{aligned}
$$

Given the values of $n$, the size of the SBN region, and the IR energy density (which is computed from the $L_{\mathrm{IR}}$ and the region size), as listed in Table 2, the expression for $B$ in the last equation (with $\eta=1$ ) can be inserted in Eq. (20), and using the other two loss rate equations, the value of $\gamma_{1}$ can be deduced. This value of $\gamma_{1}$ is then substituted in the equation for $B$ to obtain the equipartition value of the mean field. We note that in most cases the corresponding synchrotron frequency, $v_{1}=4 B \gamma_{1}^{2} \mathrm{MHz}$, where the radio spectrum is expected to curve to a flatter slope for decreasing frequencies, is comparable to our reference frequency of $5 \mathrm{GHz}$.

\section{Proton energy densities in SBNs}

In starburst galaxies SF is very intense in a relatively small nuclear region (the $\mathrm{SBN}$ ) with effective radius $r_{\mathrm{s}} \approx 0.2 \mathrm{kpc}-$ with $r_{\mathrm{s}} \equiv\left(3 / 4 R_{\mathrm{sb}}^{2} h_{\mathrm{sb}}\right)^{1 / 3}$, where $R_{\mathrm{sb}}$ and $h_{\mathrm{sb}}$ are the radius and height of the region. By contrast, low-intensity SF quiescently proceeds throughout the galactic disk (as in normal, i.e., non-starburst, spirals, such as the Galaxy).

For a sample of local SBNs, values of $U_{\mathrm{p}}$ and $B$ were calculated using the relevant observational quantities in Table 2, starting with the radio flux and spectral index. Because of appreciable observational uncertainties, mostly in the values of the gas density and the size of the emitting region, and because of modeling uncertainties, the derived values of $B$ and $U_{\mathrm{p}}$ are also uncertain, typically by a factor which we estimate to be $\sim 1.4$ and $\sim 2$. While our results for these two quantities would not seem to be that precise, it should be emphasized that some of the uncertainties are inherent given the basic difficulties in determining the size and density of the SBN, the limited spatial resolution, and the rudimentary level of the spectral $\gamma$-ray measurements. We note that, in light of these substantial uncertainties, the (modeling) uncertainty in $B$, when the latter is calculated assuming energy equipartition as compared to minimum energy, is relatively insignificant. For example, for the two well-studied nearby starburst galaxies, NGC 253 and NGC 3034 (M 82), values of $B$ are only $\sim 15 \%$ lower in the minimum energy configuration compared to the equipartition values (listed in Table 2 ). The CR-derived results are stable over much of the ISM parameter space of SBNs (Boettcher et al. 2013).

\footnotetext{
4 Energy equipartition may be attained (over time) under conditions of tight coupling of the main matter and energy components of a physical environment in its minimum energy configuration. The electromagnetic emission of a SBN that directly comes from relativistic particles manifests itself in the radio and in $\gamma$ rays. The total energy density in the SBN responsible for such nonthermal emission is $U=U_{\mathrm{CR}}+U_{B}$. As noted in Sect. 2, under physical conditions prevailing in a SBN the particles and the $B$-field behave like relativistic fluids tightly coupled with each other. Their equilibrium configuration most likely corresponds to a state of minimum energy, which is achieved for $U_{B}=\eta U_{\mathrm{CR}}$ with $\eta=3 / 4$ (Longair 1981). Clearly, this minimum-energy condition is very close to equipartition $(\eta=1)$, so at equilibrium the particle energy density nearly equals the field energy density.
}

Our electron-based estimates of the proton energy densities are in agreement with $\gamma$-ray measurements of $\pi^{0}$-decay emission for the three galaxies NGC 253, NGC 3034, and NGC 4945 for which such emission was detected. The results for the very compact SBNs of Arp 220 and Arp 299-A represent extreme cases; small source regions and relatively hard electron spectra result in high magnetic fields and high CR energy densities, $B \sim 0.2 \mathrm{mG}$ and $U_{\mathrm{p}} \sim 500 \mathrm{eV} \mathrm{cm}^{-3}$. However, it is questionable whether in such extreme environments, with conditions that are more typical of SNRs, steady state and equipartition are actually attained (e.g., Torres 2004). The unusually high values of $\gamma_{1}$ derived for these highly compact nuclei may signal, in fact, a breakdown in our assumptions, the most critical one being particle-field equipartition.

\section{Discussion}

Active SF leads to particle acceleration by SN shocks. Considerations of the acceleration, relevant energy losses, and starburst timescales, indicate that relativistic proton and electron distributions reach steady state during most of the starburst phase. Given the tight coupling between the particles and magnetic fields in the dense plasma, it is quite likely that energy equipartition is achieved in the SBN region. With an assumed theoretically motivated $\mathrm{p} / \mathrm{e}$ ratio, the assumption of equipartition provides the requisite relation to determine particle energy densities and the mean field from spectral radio measurements.

Essential to this radio-based method is a reliable estimate of the $\mathrm{p} / \mathrm{e}$ energy ratio, $\kappa$. Adopting the common assumption of an overall electrically neutral nonthermal plasma, we derived an approximate expression for this ratio as a function of the electron and proton spectral indices, $q_{\mathrm{e}}$ and $q_{\mathrm{p}}$. We note that, in the limit of $q_{\mathrm{e}}=q_{\mathrm{p}}=q(\mathrm{e} . \mathrm{g}$., at injection $), \kappa \simeq\left(m_{\mathrm{p}} / m_{\mathrm{e}}\right)^{(3-q) / 2}$; this simple relation is supplementary to the analogous, well-known $\mathrm{p} / \mathrm{e}$ number ratio, $N_{\mathrm{p}} / N_{\mathrm{e}}=\left(m_{\mathrm{p}} / m_{\mathrm{e}}\right)^{(q-1) / 2}($ e.g., Schlickeiser 2002). Even though the assumptions of single-index steady-state spectra and particle-field equipartition may be unrealistic, at our present knowledge of the SBN environment relaxing any of these simplifications necessarily leads to a more parameter-rich formalism that will invariably result in arbitrariness in guessing parameter values in what is essentially an underdetermined problem.

For the determination of $q_{\mathrm{p}}$, we have assumed that SBN $\gamma$-ray spectra (in the observed Fermi/LAT and IACT energy ranges) are dominated by emission from $\pi^{0}$-decay owing to the much higher SF rate and mean (target) gas density in the SBN than in the (rest of the) disk. The theoretical expectation of proton injection index in the range $2.0 \leq q \leq 2.3$ is fully consistent with the measured value $q_{\mathrm{p}} \simeq 2.2$. Because of the lack of spatial information on the distribution of $\gamma$-ray emission in the nearby starburst galaxies, our expectation on the respective contributions of the SBN and disk regions is based on theoretical predictions, particularly our own detailed numerical modeling of the emission in M 82 and NGC 253 (using a modified version of the GALPROP code: Persic et al. 2008 and Rephaeli et al. 2010; see also Domingo-Santamaría \& Torres 2005 for NGC 253). These analyses do suggest that while the relative contribution of the disk is not negligible, it comprises only a small fraction due to further steepening of the particle spectra in the disk. However, given the uncertainty in the exact values of both the measured and predicted power-law indices, there is also an uncertainty in the relative contributions of the SBN and disk.

Our work here improves on our earlier discussion and partial implementation of the radio-based method for determining electron and proton energy densities in active regions of SF 
(Persic \& Rephaeli 2010). We do so by accounting for electron radiative losses, by using a more accurate calculation of the $\mathrm{p} / \mathrm{e}$ ratio, and basing our approach on insight gained from our previous implementation of a modified version of the GALPROP code that fully accounts for both spectral and spatial evolution of proton and electron distributions in the SB region and throughout the disk (Persic et al. 2008; Rephaeli et al. 2010). Our general approach and its quantitative implementations for predicting the high-energy spectra of the starburst galaxies NGC 253 and NGC 3034 have been validated by good agreement with $\gamma$-ray measurements (Acero et al. 2009; Acciari et al. 2009; Ackermann et al. 2012).

Following on previous work (Beck \& Krause 2005), in a recent paper Lacki \& Beck (2013, hereafter LB13) discussed the validity of field-particle equipartition in a SB environment, accounting for secondary electrons and strong energy losses. While their main conclusions on deducing $U_{\mathrm{p}}$ from radio measurements do agree with ours (in both Persic \& Rephaeli 2010 and this work), there are substantial differences between our respective treatments. First, we start with the electron spectrum as deduced from radio measurements, including the contribution of secondary electrons to the emission. We then use an analytically derived primary $\mathrm{p} / \mathrm{e}$ energy density ratio (using the relevant parameter values) to compute $U_{\mathrm{p}}$. In contrast, the starting point of the LB13 analysis is the proton energy density, which they take to be related to the electron energy density by the same factor, 75 , for all the galaxies in their sample. Second, whereas we assume electric neutrality of the accelerated particles to determine the primary $\mathrm{p} / \mathrm{e}$ ratios for different electron and proton spectral indices, LB13 start from an assumed universal p/e number density ratio, which they adjust by accounting for the electrons' energy losses. Third, we compute the secondary-to-primary electron mumber ratio by using the primary $\mathrm{p} / \mathrm{e}$ number ratio, the mean proton free path in a gas with a given density, and the branching ratios in pp interactions, whereas LB13 estimate this ratio by scaling the injection $\mathrm{p} / \mathrm{e}$ ratio by $1 / 6$ the value of the estimated fraction of the proton energy that goes into $\pi^{ \pm}$. These differences in approach and implementation led to substantially different results for $U_{\mathrm{p}}$ and $B$ (listed in our Table 2 and their Table 3). Whatever the details, it should be noted that for the nearby starburst galaxies NGC 253, NGC 3034, and NGC 4945 our estimated $U_{\mathrm{p}}$ and $B$ agree with results of direct measurements. This agreement suggests that the equipartition assumption, which we made to derive $\mathrm{CR}$ and $B$ energy densities, is globally verified in these SBNs, at least as an average property. For NGC 3034 this suggestion was made early on by Völk et al. (1990) on the condition that $\zeta(>1 \mathrm{GeV}) \sim 10^{2}$, a condition that is consistent with our procedure, see Eq. (6).

If the agreement between radio estimates and $\gamma$-ray measurements of $U_{\mathrm{p}}$ and B in SBNs is further validated and established, the radio method for reliable estimation of proton energy densities will be particularly useful for distant $(z \gtrsim 1)$ galaxies whose intense SF is exemplified (albeit at lower levels) by the nearby starburst galaxies, but whose faint $\gamma$-ray fluxes are not detectable with current or upcoming instruments, whereas their $<0.1 \mathrm{mJy}$ radio fluxes are (e.g., Tozzi et al. 2009).

\section{References}

Acciari, V. A., Aliu, E., et al. (VERITAS Collaboration) 2009, Nature, 462, 770 Acero, F., Aharonian, F., Akhperjanian, A. G., et al. (H.E.S.S. Collaboration) 2009 Science, 326, 1080

Ackermann, M., Ajello, M., Allafort, A., et al. (LAT Collaboration) 2012, ApJ, 755, A164
Baltrusaitis, R. M., Cassiday, G. L., Elbert, J. W., et al. 1984, Phys. Rev. Lett., 52,1380

Beck, R., \& Krause, M., 2005, Astron. Nachr., 326, 414

Blumenthal, G. R., \& Gould, R. J. 1970, Rev. Mod. Phys., 42, 237

Boettcher, E., Zweibel, E. G., Yoast, T. M., \& Gallagher, J. S. III 2013, ApJ, 779, 12

Bondi, M., Perez-Torres, M. A., Herrero-Illana, R., \& Alberdi, A. 2012, ApJ, 539, A134

Brock, D., Joy, M., Lester, D. F., Harvey, P. M., \& Ellis, H. B., Jr. 1988, ApJ, 329, 208

Calzetti, D., Conselice, Ch. J., Gallagher, J. S., \& Kinney, A. L. 1999, AJ, 118, 797

Carlstrom, J. E., \& Kronberg, Ph. P. 1991, ApJ, 366, 422

Charmandaris, V., Stacey, G. J., \& Gull, G. 2002, ApJ, 571, 282

Carral, P., Hollenbach, D. J., Lord, S. D., et al. 1994, ApJ, 423, 223

Chevalier, R. A., \& Clegg, A. W. 1985, Nature, 317, 44

Condon, J. J., Condon, M. A., Gisler, G., \& Puschell, J. J. 1982, ApJ, 252, 102

Dahlem, M., Heckman, T. M., Fabbiano, G., Lehnert, M. D., \& Gilmore, D. 1996, ApJ, 461, 724

de Cea Del Pozo, E., Torres, D. F., \& Rodriguez Marrero, A. Y., 2009, ApJ, 698, 1054

Domingo-Santamaría, E., \& Torres, D. F. 2005, A\&A, 444, 403

Drury, L. O'C., Aharonian, F. A., \& Völk, H. J. 1994, A\&A, 287, 959

Elmouttie, M., Haynes, R. F., Jones, K. L., et al. 1997, MNRAS, 284, 830

Engelbracht, C. W., Rieke, M. J., Rieke, G. H., \& Latter, W.B. 1996, ApJ, 467, 227

Engelbracht, C. W., Rieke, M. J., Rieke, G. H., Kelly, D. M., \& Achtermann, J M. 1998, ApJ, 505, 639

Fujita, Y., Ohira, Y., Tanaka, S. J., \& Takahara, F. 2009, ApJ, 707, L179

Gaisser, T. K. 1990, Cosmic Rays and Particle Physics (Cambridge: Cambridge University Press)

Gould, R. J. 1975, ApJ, 196, 689

Heckman, T. M., \& Lehnert, M. D. 2000, ApJ, 537, 690

Israel, F. P., \& Baas, F. 2001, A\&A, 371, 433

Israel, F. P., Baas, F., \& Maloney, P. R. 1990, A\&A, 237, 17

Klein, U., Wielebinski, R., \& Morsi, H. W. 1988, A\&A, 190, 41

Krabbe, A. C., Rosa, D. A., Dors O. L., Jr., et al. 2014, MNRAS, 437, 1155

Lacki, B. C., \& Beck, R. 2013, MNRAS, 430, 3171

Lenain, J.-P., Ricci, C., Türler, M., Dorner, D., \& Walter, R. 2010, A\&A, 524, L72

Lipari, S., Tsvetanov, Z., \& Macchetto, F. 1997, ApJSS, 111, 369

Longair, M. S. 1981, High Energy Astrophysics, 1st ed. (Cambridge: Cambridge Univ. Press)

Murphy, E. J., Condon, J. J., Schinnerer, E., et al. 2011, ApJ, 737, 67

Paglione, T. A. D., Marscher, A. P., Jackson, J. M., \& Bertsch D. L. 1996, ApJ, 460, 295

Persic, M., \& Rephaeli, Y. 2010, MNRAS, 403, 1569

Persic, M., \& Rephaeli, Y. 2014, in Proc. 13th Marcel Grossmann Meeting, eds. R. T. Jantzen, K. Rosquist, \& R. Ruffini (Singapore: World Scientific), in press [arXiv: 1405. 3107]

Persic, M., Rephaeli, Y., \& Arieli, Y. 2008, A\&A, 486, 143

Petuchowski, S. J., Bennett, C. L., Haas, M. R., et al. 1994, ApJ, 427, L17

Rephaeli, Y. 1979, ApJ, 227, 364

Rephaeli, Y., Arieli, Y., \& Persic, M. 2010, MNRAS, 401, 473

Rickard, L. J., Palmer, P., Morris, M., Turner, B. E., \& Zuckerman, B. 1977, ApJ, 213,673

Rieke, G. H., Lebofsky, M. J., Thompson, R. I., Low, F. J., \& Tokunaga, A. T. 1980, ApJ, 238, 24

Schinnerer, E., Böker, T., Emsellen, E., \& Lisenfeld, U. 2006, ApJ, 649, 181

Schlickeiser, R. 2002, in Cosmic Ray Astrophysics (Berlin: Springer), 472

Spoon, H. W. W., Koornneef, J., Moorwood, A. F. M., Lutz, D., \& Tielens, A. G. G. M. 2000, A\&A, 357, 898

Strickland, D. K., \& Heckman, T. M. 2009, ApJ, 697, 2030

Sugai, H., Davies, R. I., Malkan, M. A., et al. 1999, ApJ, 527, 778

Sukumar, S., Klein, U., \& Gräve, R. 1987, A\&A, 184, 71

Torres, D. F. 2004, ApJ, 617, 966

Tozzi, P., Mainieri, V., Rosati, P., et al. 2009, ApJ, 698, 740

Völk, H. J., Klein, U., \& Wielebinski, R. 1990, A\&A, 237, 21

Völk, H. J., Aharonian, F. A., \& Breitschwerdt, D. 1996, Space Sci. Rev., 75, 279

Weiss, A., Neininger, N., Hüttemeister, S., \& Klein, U. 2001, A\&A, 365, 571

Yun, M. S., Scoville, N. Z., \& Shukla, H. 2004, in The Neutral ISM in Starburst Galaxies, eds. S. Aalto, S. Hüttermeister, \& A. Pedlar, ASP Conf. Ser., 320, 27

Zhao, J.-H., Anantharamaiah, K. R., Goss, W. M., \& Viallefond, F. 1997, ApJ, 482,186 